\title{
Malignant fibrothecomatous tumour of the ovary: diagnostic value of anti-inhibin immunostaining
}

\author{
W G McCluggage, J M Sloan, D D Boyle, P G Toner
}

\begin{abstract}
Malignant ovarian tumours of the fibrothecoma group are rare. The clinicopathological features of a case of ovarian malignant fibrothecoma in which there was metastatic disease in the small intestine and peritoneum at presentation are described. A number of differential diagnoses were considered but positive immunohistochemical staining of the resected ovarian and small intestinal neoplasms with anti-inhibin was of value in confirming a sex cord-stromal tumour and in excluding other lesions. The two tumours were also ultrastructurally identical. Classical malignant fibrothecomas are said to show four or more mitotic figures per 10 high power fields (HPF). Although the intestinal secondary was mitotically active, the primary ovarian tumour contained only one to two mitoses per $10 \mathrm{HPF}$, showing that formal mitotic counts are not an absolute indicator of malignant behaviour in this group of tumours. (f Clin Pathol 1998;51:868-871)
\end{abstract}

Keywords: ovarian tumour; fibrothecoma; inhibin; immunohistochemistry

Malignant ovarian tumours of the fibrothecoma group are exceedingly rare..$^{1-4}$ They are generally classified as fibrosarcomas and there is doubt as to whether a true malignant form of thecoma exists. The main histological feature said to be of importance in distinguishing a cellular fibrothecoma from fibrosarcoma is the degree of mitotic activity in the primary tumour. ${ }^{2}$ In this report we describe an ovarian fibrothecomatous tumour with metastatic disease in the small intestine and peritoneum. Both the primary ovarian neoplasm and the intestinal metastasis were histologically and ultrastructurally indistinguishable from a cellular fibrothecoma. There were only scattered mitotic figures in the ovarian lesion, although the intestinal secondary was more mitotically active. A number of differential diagnoses were considered. Positive immunohistochemical staining of both lesions with anti-inhibin assisted in classifying the neoplasm as a malignant ovarian sex cord-stromal tumour.

\section{Case report}

A 61 year old postmenopausal woman presented with a three month history of general malaise and melaena. Rectal examination suggested a pelvic mass and she was referred to a gynaecologist. The presence of a large mass filling the pelvis was confirmed by transvaginal ultrasound scan, which also revealed free fluid in the pelvic cavity. Serum CA-125 was markedly raised at $196 \mathrm{U} / \mathrm{ml}$. A presumptive diagnosis of ovarian cancer was made. At laparotomy, tumour masses were present in the right ovary and in the terminal ileum. There were also multiple tumour nodules throughout the abdominal peritoneum. The clinical impression was of a primary lesion in the right ovary, with small intestinal and peritoneal metastases. Total abdominal hysterectomy and bilateral salpingo-oophorectomy was performed, together with resection of a length of terminal ileum. The postoperative period was unremarkable and the patient is currently undergoing chemotherapy.

\section{Methods}

The surgical specimen was fixed in formalin and sections for histological examination were routinely processed in paraffin wax and stained with haematoxylin and eosin. Reticulin and oil-red O stains were performed on representative sections of the ovarian and small intestinal tumours. A formal mitotic count was carried out on all histological sections of the ovarian and small intestinal neoplasms. This was done by counting the number of mitotic figures in 50 high power fields ( $\mathrm{HPF}$ ) and calculating the average per $10 \mathrm{HPF}$.

Immunohistochemistry was performed using a standard streptavidin biotin-peroxidase method (Dako). Sections were stained with the following monoclonal antibodies: inhibin (Serotec), vimentin (Dako), CAM 5.2 (Becton Dickinson), CA-125 (CIS Biointernational), desmin (Dako), S-100 protein (Diagnostic Products), and $\alpha$ smooth muscle actin (Sigma). The anti-inhibin antibody is a mouse monoclonal antibody against the $\alpha$ subunit of human inhibin. Immunohistochemistry was performed using appropriate positive and negative controls. For anti-inhibin staining, positive controls comprised ovaries containing follicular cysts or corpora lutea. For negative controls, the primary antiserum was replaced by mouse immunoglobulin (IgG, Dako) at a comparable protein concentration. For comparison, three cases each of leiomyosarcoma and gastrointestinal stromal tumour were also stained with anti-inhibin.

Representative pieces of formalin fixed tissue from both the ovarian and small intestinal tumours were processed for examination by electron microscopy. Ultrathin sections were stained with uranyl acetate and lead citrate.
Accepted for publication 23 June 1998 

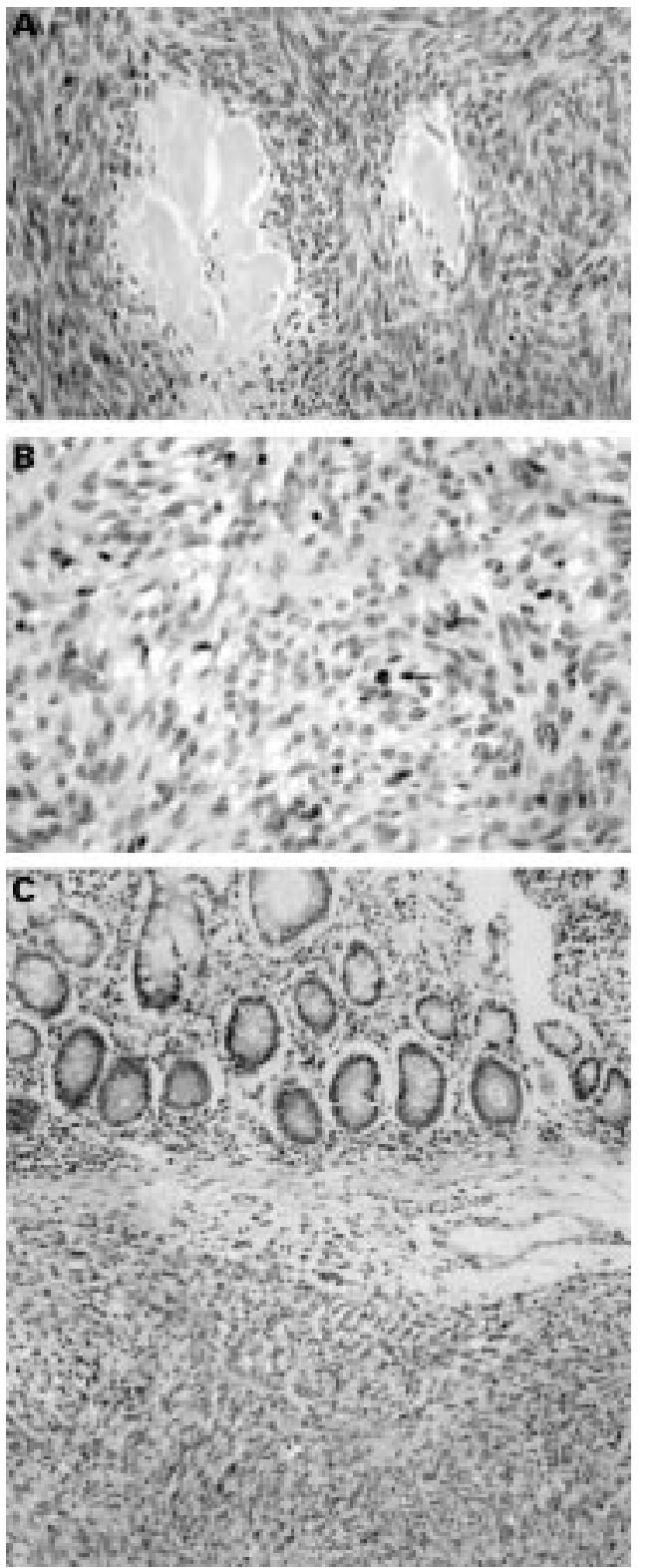

Figure 1 (A) Ovarian tumour showing a cellular spindle cell lesion with hyalinised plaques. (B) Higher power of ovarian tumour. An occasional mitotic figure (arrow) is present. (C) Metastatic lesion in small intestine. Tumour is situated under the mucosal surface.

\section{Results}

PATHOLOGICAL FINDINGS

The surgical specimen consisted of a uterus and cervix with attached ovaries and fallopian tubes. An $8 \mathrm{~cm}$ length of small intestine was also received. The right ovary was replaced by a solid, yellow coloured tumour which weighed $80 \mathrm{~g}$ and measured $7 \mathrm{~cm}$ in maximum diameter. The left ovary weighed $6 \mathrm{~g}$ and measured $3 \mathrm{~cm}$ in maximum diameter. It was grossly unremarkable. The uterus contained two intramural fibroids, both measuring $1 \mathrm{~cm}$ in maximum diameter. A $9 \mathrm{~cm}$ diameter partially necrotic tumour was present in the small intestine. This was mainly located on the serosal surface, but infiltrated the wall and focally ulcerated the mucosa.

Histological examination of multiple sections from the tumour in the right ovary showed a cellular lesion. Tumour cell nuclei were ovoid to spindle shaped and contained evenly dispersed chromatin (fig 1A). Neither nuclear grooves nor Call-Exner bodies were identified and there was little nuclear pleomorphism. Numerous hyalinised plaques were present and there were areas of oedema. There was no haemorrhage or necrosis. Scattered mitotic figures were identified (fig 1B), a formal mitotic count revealing one to two per $10 \mathrm{HPF}$. The reticulin stain revealed a pericellular arrangement of fibres and numerous cytoplasmic lipid droplets were seen with the oil-red $\mathrm{O}$ stain. The histological features were in keeping with a cellular fibrothecoma.

Histological examination of the left ovary showed a few microscopic foci of tumour, similar to that in the right ovary, on the external surface. In areas, tumour cells had abundant eosinophilic cytoplasm, in keeping with luteinisation. Given their multifocality and location on the external surface, these were presumed to represent metastatic disease rather than independent primary tumours. The fallopian tubes were unremarkable. The endometrium showed definite proliferative activity with nuclear stratification and mitotic activity. However, there was no hyperplasia or malignancy. The presence of two benign intramural fibroids was confirmed. No microscopic abnormality was identified within the cervix.

Histological examination of multiple sections from the tumour in the small intestine confirmed that it was mainly located on the serosal surface, but also invaded the muscularis propria and submucosa and focally ulcerated the mucosa (fig 1C). There were areas of haemorrhage and necrosis. Tumour cell nuclei were similar to those in the right ovarian neoplasm, but focally there was a much higher mitotic rate, a formal mitotic count revealing areas in which there were $12-15$ mitoses per 10 HPF. Several microscopic foci of metastatic tumour were identified adjacent to the main secondary tumour mass.

IMMUNOHISTOCHEMICAL FINDINGS

The immunophenotypes of the right ovarian and small intestinal neoplasms were identical. There was positive cytoplasmic staining with anti-inhibin (fig 2) and vimentin, but no staining with the other antibodies employed. The three leiomyosarcomas and the three gastrointestinal stromal tumours were all negative with anti-inhibin.

\section{ELECTRONMICROSCOPY}

Ultrastructural examination of the ovarian and small intestinal tumours showed identical features. Tumour cells contained ovoid to spindle shaped nuclei and a moderate amount of cytoplasm. Lipid droplets, both intracellular and extracellular, were easily identified. Poorly formed adhesion specialisations were present between adjacent cells. A discontinuous external lamina appeared at the cell surface in some areas and there were scattered collagen fibrils in the interstitium. There was no ultrastructural evidence of smooth muscle or neural differentiation. 


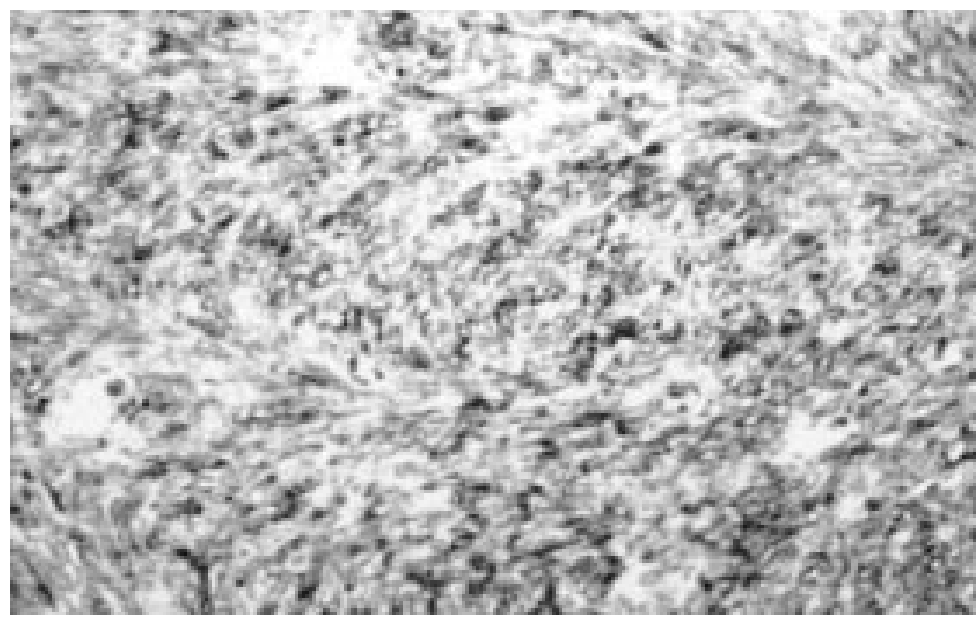

Figure 2 Positive immunohistochemical staining of the ovarian tumour with anti-inhibin.

\section{Discussion}

Ovarian tumours of the fibrothecoma group are relatively common. Along with granulosa cell tumour they belong to the group of sex cord-stromal neoplasms. The vast majority of fibrothecomas behave in a benign fashion and malignant variants are exceedingly rare..$^{-4}$ Occasional cases of malignant thecoma have been reported, ${ }^{4}$ but there is doubt as to whether a true malignant variant of this neoplasm exists. In a critical review of published reports on malignant thecoma, Waxman et al concluded that most of the recorded cases probably represented sarcomatoid adult granulosa cell tumour, stromal sarcoma, or fibrosarcoma, and that if a thecoma ever becomes malignant, tumour cells dedifferentiate so that they can no longer be recognised as theca cells. ${ }^{4}$ They proposed that the term malignant thecoma should not be used.

Tumours arising from the ovarian stroma comprise a spectrum ranging from typical fibroma at one end to typical thecoma at the other. Many tumours have an intermediate histological appearance and in surgical pathology practice are often not separated, but rather categorised as fibrothecoma. The histological features of the right ovarian tumour in the present case are entirely in keeping with a cellular fibrothecoma. Typical features include the presence of hyaline plaques, the pericellular arrangement of reticulin fibres, the presence of lipid droplets, and the occurrence of foci where tumour cells contained abundant eosinophilic cytoplasm, in keeping with luteinisation. Although there was no evidence of endometrial hyperplasia, the endometrium showed definite proliferative activity, suggesting an oestrogenic effect in this postmenopausal woman. The presence of lipid droplets and of endometrial proliferative activity suggests that the neoplasm may justifiably be categorised as a thecoma although, as already stated, we prefer the designation fibrothecoma.

Prat and Scully described the clinicopathological features of 17 cases of obviously malignant as well as cellular fibromatous tumours of the ovary. ${ }^{2}$ They concluded that the tumours fell into two categories: a usually benign form with one to three mitotic figures per $10 \mathrm{HPF}$, designated cellular fibroma, and a malignant form with four or more mitoses per $10 \mathrm{HPF}$, designated fibrosarcoma. Nuclear pleomorphism and other indices were found to be much less reliable indicators of malignancy. Using the criteria of Prat and Scully, the primary tumour within the right ovary in our case would have been classified as a cellular fibrothecoma. This shows that mitotic counts are not an absolute indicator of malignancy in this group of neoplasms. Lyday describes an interesting case of ovarian fibroma with abdominal implants, all of which were histologically benign. ${ }^{5}$ The patient was alive and clinically free of tumour three years after diagnosis.

Although the histological appearance of the right ovarian tumour was characteristic of a cellular fibrothecoma, the appearance of the concurrent small intestinal neoplasm led us to consider other possible diagnoses, including leiomyosarcoma and gastrointestinal stromal tumour. It was uncertain whether the two neoplasms were related, or whether they represented separate primary tumours. Immunohistochemistry was helpful-the immunophenotype of the two neoplasms was identical, both staining positively with an antibody against inhibin. Inhibin is a peptide hormone which is normally produced by ovarian granulosa cells and which inhibits the release of follicle stimulating hormone from the pituitary gland, thus acting as a modulator of folliculogenesis. ${ }^{6}$ It is composed of an $\alpha$ subunit and a $\beta$ subunit. Recently, immunohistochemical staining with anti-inhibin has been performed on tissue sections, and the antibodies (especially against the $\alpha$ subunit) have been found to be good markers of ovarian sex cord-stromal tumours. ${ }^{7}$ Although mostly investigated in granulosa cell tumours, positivity has also consistently been demonstrated in fibrothecomas and other sex cord-stromal tumours. ${ }^{78}$ Positive staining with antibodies against $\alpha$ inhibin assist in confirming a sex cord-stromal tumour and in excluding other lesions which may enter into the differential diagnosis, including carcinomas with a sex cord pattern. Leiomyomatous tumours have been reported to be negative. ${ }^{8}$ In addition, we stained three cases each of leiomyosarcoma and gastrointestinal stromal tumour with antiinhibin and found no immunoreactivity.

In the present case, the absence of staining for desmin and $\alpha$ smooth muscle actin helped to exclude a leiomyosarcoma, as did electronmicroscopy, which showed no evidence of smooth muscle differentiation. Fibrothecomatous tumours have no unique ultrastructural markers, but the features of the present tumour were consistent with previous ultrastructural descriptions of typical cases $^{4}$ and did not suggest any alternative diagnosis. The presence of adhesion specialisations between tumour cells and of basal lamina-type material was perhaps slightly unusual, but these features have been described in ovarian fibrothecomas. ${ }^{9}$ A diffuse or sarcomatoid variant of adult granulosa cell tumour was also considered. Immunoreactivity with anti-inhibin would have been in keeping with this diagnosis, but adult granulosa cell tumour was considered unlikely 
owing to the absence of the characteristic nuclear grooving and of Call-Exner bodies. In addition, the presence of hyalinised plaques and the pericellular arrangement of reticulin fibres was more in keeping with a fibrothecomatous neoplasm than an adult granulosa cell tumour, which is generally not fibrilloformative.

The preoperative serum CA-125 concentration was markedly increased, resulting in clinical suspicion of ovarian cancer. The CA-125 antigen, initially described as a marker of nonmucinous ovarian neoplasms, is expressed on the surface of mesothelial cells. ${ }^{10}$ Raised serum concentrations of CA-125 have been found in a variety of non-ovarian malignancies and in non-neoplastic conditions such as endometriosis and cirrhosis. It has been postulated that these raised levels may be caused by peritoneal irritation by ascites, tumour infiltration, or various other factors. In the present case, the multiple metastatic tumour nodules on the abdominal peritoneum may have been responsible for CA-125 production by mesothelial cells. There was no staining of the primary or secondary tumour with an antibody against CA-125.

In conclusion, we report the clinicopathological features of a case of ovarian malignant fibrothecoma and illustrate the value of antiinhibin staining in establishing a diagnosis of sex cord-stromal tumour.

1 Miles PA, Kiley KC, Mena H. Giant fibrosarcoma of the ovary. Int f Gynecol Pathol 1985;4:83-7.

2 Prat J, Scully RE. Cellular fibromas and fibrosarcomas of the ovary: a comparative clinicopathologic analysis of seventeen cases. Cancer 1981;47:2663-70.

3 Tsuji T, Kawauchi S, Utsunomiya T, et al. Fibrosarcoma verses cellular fibroma of the ovary. A comparative study of their proliferative activity and chromosome aberrations using MIB1 immunostaining, DNA flow cytometry, and fluorescence in situ hybridization. Am $\mathscr{f}$ Surg Pathol 1997;21:52-9.

4 Waxman M, Vuletin JC, Urcuyo R, et al. Ovarian low-grade stromal sarcoma with thecomatous features. A critical reappraisal of so-called "malignant thecoma". Cancer 1979;44:2206-17.

5 Lyday RO. Fibroma of the ovary with abdominal implants. Am ₹ Surg 1952;84:737-738.

6 McLachlan RI, Robertson DM, Burger HG, et al. Circulating immunoreactive inhibin levels during the normal menstrual cycle. f Clin Endocrinol Metab 1987;65:954-61.

7 Flemming P, Wellmann A, Maschek H, et al. Monoclonal antibodies against inhibin represent key markers of adult granulosa tumors of the ovary even in their metastases. Am f Surg Pathol 1995;19:927-33.

8 McCluggage WG, Maxwell P, Sloan JM. Immunohistochemical staining of ovarian granulosa cell tumors with . 1034-8.

9 Erlandson RA. Ultrastructural features of specific human neoplasms with clinicopathologic, immunohistochemical and cytogenetic correlations. In: Erlandson RA, ed. Diagnostic transmission electron microscopy of tumours, 1 st ed. New York: Raven Press, 1994:375-8.

10 Nouwen EJ, Pullet DE, Edekens MW, et al. Immunohistochemical localisation of placental alkaline phosphatase, carcinoembryonic antigen, and cancer antigen 125 in normal and neoplastic human lung. Cancer Res 1986;46:866-76. 